\title{
Interactions and star formation
}

\section{Johan H. Knapen $^{1,2}$, Mauricio Cisternas ${ }^{1,2}$ and Miguel Querejeta ${ }^{3}$}

\author{
${ }^{1}$ Instituto de Astrofísica de Canarias, E-38205 La Laguna, Tenerife, Spain \\ ${ }^{2}$ Departamento de Astrofísica, Universidad de La Laguna, E-38205 La Laguna, Tenerife, Spain \\ ${ }^{3}$ Max Planck Institute for Astronomy, Königstuhl 17, 69117, Heidelberg, Germany
}

\begin{abstract}
We investigate the influence of interactions on the star formation by studying a sample of almost 1500 of the nearest galaxies, all within a distance of $\sim 45 \mathrm{Mpc}$. We define the massive star formation rate (SFR), as measured from far-IR emission, and the specific star formation rate (SSFR), which is the former quantity normalized by the stellar mass of the galaxy, and explore their distribution with morphological type and with stellar mass. We then calculate the relative enhancement of these quantities for each galaxy by normalizing them by the median SFR and SSFR values of individual control populations of similar non-interacting galaxies. We find that both SFR and SSFR are enhanced in interacting galaxies, and more so as the degree of interaction is higher. The increase is, however, moderate, reaching a maximum of a factor of 1.9 for the highest degree of interaction (mergers). The SFR and SSFR are enhanced statistically in the population, but in most individual interacting galaxies they are not enhanced at all. We discuss how those galaxies with the largest SFR and/or SSFR enhancement can be defined as starbursts. We argue that this study, based on a representative sample of nearby galaxies, should be used to place constraints on studies based on samples of galaxies at larger distances.
\end{abstract}

Keywords. Galaxies: general, Galaxies: interactions, Galaxies: starbursts

\section{Introduction}

Galaxy-galaxy interactions and mergers are an important factor in the dominant cosmological model of galaxy evolution, in which mergers between dark matter haloes and/or luminous galaxies occur frequently, increasing their mass and ultimately shaping them into the galaxies that we observe at the current epoch. A frequent assumption is that interactions and mergers lead to star formation rates (SFRs) that are temporarily increased, often by very large amounts. We know from observations that this can and does happen, in the most extreme form as Ultra-Luminous InfraRed Galaxies (ULIRGs), and numerical simulations can also produce much enhanced SFRs (e.g., Joseph \& Wright 1985; Barnes \& Hernquist 1991).

A question that is vital but has not been answered is whether any galaxy-galaxy interaction or merger is accompanied by a significant increase in the SFR, and whether this increase is always so large that is can be classified as a so-called 'starburst'. From various observational and modeling studies we know that indeed there is such a general increase, but also that this is limited to around a factor of two in the SFR (e.g., Larson \& Tinsley 1978; Hernández-Toledo et al. 2005; Di Matteo et al. 2007; Knapen \& James 2009; Ellison et al. 2013; Knapen, Cisternas \& Querejeta 2015 and references therein).

We summarise here the results of our statistical study of the SFR and the specific SFR (SSFR; SFR normalized by stellar mass) of a sample of some 1500 galaxies in the very local Universe, within a distance of $\sim 45 \mathrm{Mpc}$ (Knapen et al. 2015; Knapen \& Cisternas 2015). This sample is unique because it covers galaxies down to stellar masses of around $10^{8} M_{\odot}$ and of all morphological types, and should be considered as a local analogue for studies of galaxies at higher redshifts. 

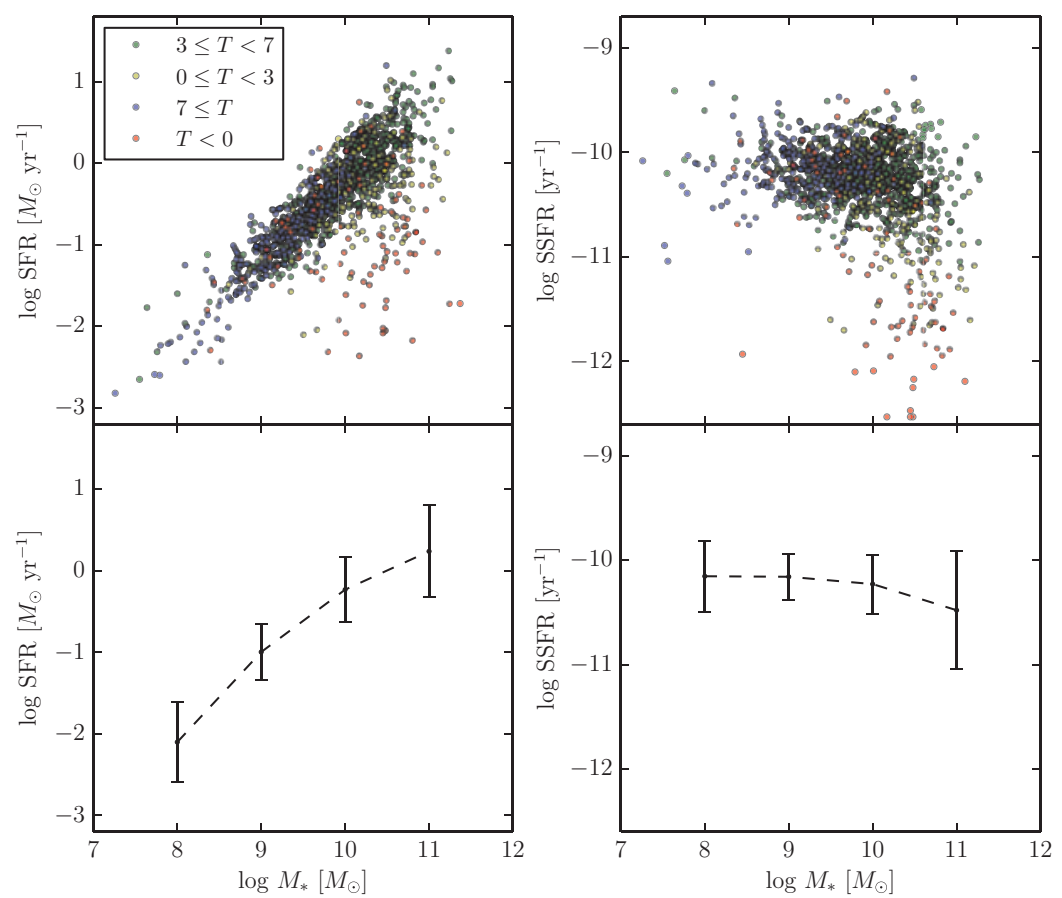

Figure 1. Log of the SFR (left panels) and the SSFR (right panels) as a function of stellar mass. Top panels show the individual galaxies, colour-coded by morphological type (for reference, red roughly corresponds to Hubble types E and S0; yellow, to early-type spirals; green, to late-type spirals; blue, to irregulars), while the lower panels show the median values, with the $1 \sigma$ spread indicated by the error bars. The dashed curve connects the points. From Knapen et al. (2015).

\section{Data and Analysis}

For a sample of 1478 nearby galaxies $(D<45 \mathrm{Mpc})$ selected from the Spitzer Survey of Stellar Structure in Galaxies ( $\mathrm{S}^{4} \mathrm{G}$; Sheth et al. 2010) we use the SFR (from a combination of IRAS 60 and $100 \mu \mathrm{m}$ fluxes; from Querejeta et al. 2015), the SSFR (SFR divided by stellar mass, the latter from the dust emission-corrected Spitzer $3.6 \mu \mathrm{m}$ images from the $\mathrm{S}^{4} \mathrm{G}$; also from Querejeta et al. 2015), and whether the galaxies are interacting (classes A - merging, B - highly distorted, C-with minor distortions; from Knapen et al. 2014).

We then calculate, for each sample galaxy, the enhancement in its SFR and SSFR, $E$ (SFR) and $E$ (SSFR), by dividing these parameters by the median values for a control sample. The control sample is created for each galaxy individually, and consists of all those galaxies which are not interacting and which are close in morphological type (within \pm 1 numerical class) and stellar mass (within \pm 0.2 in $\log \left(M / M_{\odot}\right)$; see Knapen et al. 2015 ).

We can use the values of $E(\mathrm{SFR})$ and $E(\mathrm{SSFR})$ to define which galaxies are starbursts: namely those which have values of $E$ above a certain level, in particular $E(\mathrm{SFR})>5$ and $E(\mathrm{SSFR})>4$. As we show in Knapen et al. (2015; see also Knapen \& James 2009), these choices are reasonable, but in any case the main results obtained do not depend on the exact values of the cut-off used to define starbursts.

\section{Results}

In Figure 1 we show how the SFR and the SSFR vary with stellar mass and with morphological type (the latter colour-coded in the top panels). This is a well-known 

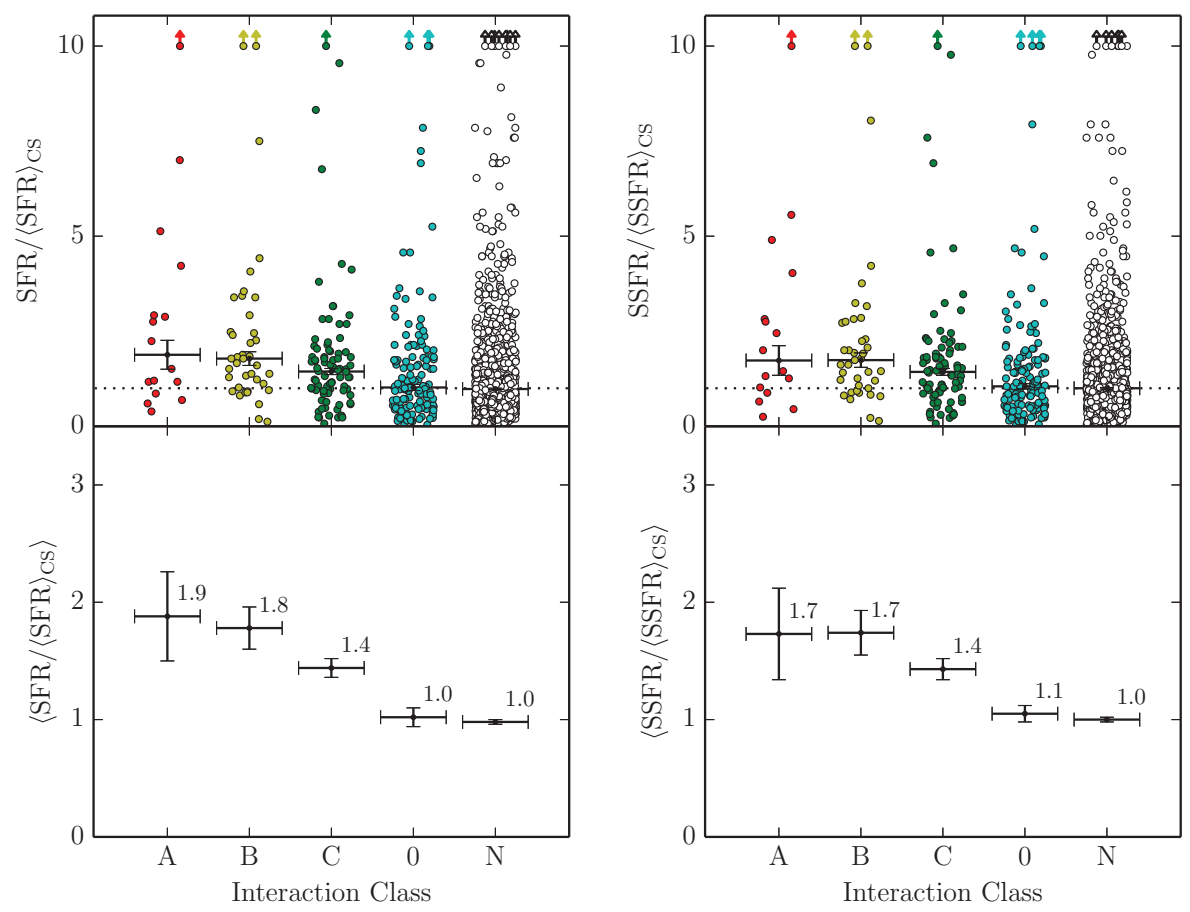

Figure 2. The SFR (left panels) and SSFR (right panels) enhancement, defined as the (S)SFR of a galaxy normalized by the median (S)SFR for its control population, separated by interaction class (where $\mathrm{A}$ is the most extreme class, of mergers, and $\mathrm{N}$ contains non-interacting galaxies). Values larger than 10 are indicated by lower limits. Median values per class are indicated with their $1 \sigma$ uncertainty in both top and lower panels, but are amplified in the lower panels where the values are indicated next to the data points. From Knapen et al. (2015). A color version of this figure is available online.

plot (often referred to as the "galaxy main sequence") but nicely illustrates a number of important points, such as that our (S)SFR determinations seem correct, and that our sample spans a large range in both morphological type and mass. We claim that our sample is a much better representation of at least the nearby Universe than what a typical galaxy main-sequence plot from the literature might lead one to believe.

Figure 2 shows the enhancement of the SFR (left panels) and the SSFR (right panels) for each galaxy individually (top panels) and in the median (lower panels) for the sample galaxies as a function of their interaction class (see Sect. 2), where the most strongly interacting galaxies are category $\mathrm{A}$ on the left, and the non-interacting control sample galaxies are class $\mathrm{N}$ on the right. We can derive a number of main results from this figure.

(a) From the individual values (top panels) we see that extreme values of SFR and SSFR, of more than 10 times that of the control sample, occur across the board, in all categories. These are not limited to interacting galaxies.

(b) Values below unity also occur across the board, and in particular we note that a good number of interacting and merging galaxies (classes C, B and A) form stars at a lower rate than might be expected from their mass and type. Many interacting galaxies have no enhanced (S)SFR at all.

(c) From the median SFR and SSFR enhancement values (lower panels) we see that there is indeed an increase in (S)SFR in interacting as compared to non-interacting (class $\mathrm{N}$ ) galaxies, but that this enhancement is modest, just below a factor of two. 
(d) This increase in (S)SFR is more pronounced as the interaction gets stronger, towards class A or towards the left in the plots.

(e) Finally, we see that our control sample technique works: the median values for class $\mathrm{N}$ (the non-interacting galaxies, each of which has also been analyzed with its own control sample) are 1.0, as they should be.

\section{Conclusions}

Using a sample of 1500 nearby galaxies from the $S^{4} \mathrm{G}$ we quantify the role of galaxygalaxy interactions in enhancing the star formation. To do this, we measure SFRs from IRAS fluxes, and use stellar masses determined from $3.6 \mu \mathrm{m} \mathrm{S}{ }^{4} \mathrm{G}$ images corrected for dust emission. We normalize the SFR by galaxy mass (yielding the SSFR), and explore the distribution of the SFR and SSFR with morphological type and galaxy stellar mass. For each galaxy we calculate the SFR and SSFR enhancement by normalizing them to the values obtained for a specific and individually constructed control sample, consisting of all galaxies within a morphological type range of \pm 1 , and a stellar mass range of \pm 0.2 in $\log \left(M / M_{\odot}\right)$.

We find that both SFR and SSFR are enhanced in interacting galaxies, and more so as the interaction becomes stronger. The increase is, however, moderate, of at most a factor of two. We can use the SFR and SSFR enhancements to define galaxies as starbursts. The largest values of (S)SFR enhancement, indicative of the most extreme starbursts, occur in all classes, not just among the interacting galaxies. Many interacting galaxies have SFRs and SSFRs that are not enhanced at all with respect to a control population, and in several cases their (S)SFR is even lower. As this study is based on a representative sample of nearby galaxies, we argue that it should be used to place constraints on studies based on samples of galaxies at larger distances.

\section{Acknowledgments}

We acknowledge support to the DAGAL network from the People Programme (Marie Curie Actions) of the European Union's Seventh Framework Programme FP7/2007-2013/ under REA grant agreement PITN-GA-2011-289313, and from the Spanish Ministry of Economy and Competitiveness (MINECO) under grant number AYA2013-41243-P.

\section{References}

Barnes, J. E. \& Hernquist, L. E. 1991, ApJ, 370, L65

Di Matteo, P., Combes, F., Melchior, A.-L., \& Semelin, B. 2007, A\&A, 468, 61

Ellison, S. L., Mendel, J. T., Patton, D. R., \& Scudder, J. M. 2013, MNRAS, 435, 3627

Hernández-Toledo, H. M., Avila-Reese, V., Conselice, C. J., \& Puerari, I. 2005, AJ, 129, 682

Joseph, R. D. \& Wright, G. S. 1985, MNRAS, 214, 87

Knapen, J. H. \& Cisternas, M. 2015, it ApJL, 807, L16

Knapen, J. H., Cisternas, M., \& Querejeta, M. 2015, MNRAS, 454, 1742

Knapen, J. H., Erroz-Ferrer, S., Roa, J., et al. 2014, A\&A, 569, AA91

Knapen, J. H. \& James, P. A. 2009, ApJ, 698, 1437 (KJ09)

Larson, R. B. \& Tinsley, B. M. 1978, ApJ, 219, 46

Querejeta, M., Meidt, S. E., Schinnerer, E., et al. 2015, ApJS, 219, 5

Sheth, K., Regan, M., Hinz, J. L., et al. 2010, PASP, 122, 1397 